\title{
Evaluation the Fatigue crack Closure in Metal using Eddy Current Pulsed Thermography
}

Jianping Peng, Lingfan Feng, Jie Bai, Hui Yin, Jianji Fu, Jianguang Guo, Zhu He*

School of Physical Science and Technology, Southwest Jiaotong University, Chengdu 610031, PR China

\begin{abstract}
:
Detection of crack is important for the evaluation of material condition in railway wheel tread.

If a fatigue crack is closed because of residual stress or loading, the crack could be underestimated or overlooked. To simulate the fatigue crack in railway, some deep fatigue cracks in the specimens are detected and imaged by Eddy Current Pulsed Thermography (ECPT) with various crack propagation length. Some features are extracted and studied also to quantify the crack closure. The proposed method greatly enhances the capability for cracks detection and evaluation.
\end{abstract}

Keywords: Eddy current pulsed thermography, crack closure, residual stress, railway

This work is supported by the National Natural Science Foundation (No. 61771409) and Sichuan natural foundation (No.2018GZ20047).

Reference:

[1] J. Peng, G. Y. Tian, L. Wang, Y. Zhang, K. Li, and X. Gao, "Investigation into eddy current pulsed thermography for rolling contact fatigue detection and characterization," NDT \& E International, vol. 74, pp. 72-80, 9,2015.

[2] SCHIEFELBEIN B E, HOLLAND S D, BASTAWROS A. Precision measurement of crack closure state with vibrothermography [J]. 2016, 1706(1): 473-80.

[3] Ohara Y, Takahashi K, Ino Y, et al. High-selectivity imaging of closed cracks in a coarsegrained stainless steel by nonlinear ultrasonic phased array[J]. Ndt \& E International, 2017, 91. 